\title{
Dose de esterco de ave poedeira e suprimento de nitrogênio à cultura do trigo ${ }^{1}$
}

\author{
\begin{tabular}{l}
\hline Enrique A. Figueroa ${ }^{2}$, Pedro A. V. Escosteguy ${ }^{3} \&$ Sirio Wiethölter \\
\hline
\end{tabular}
}

\section{RESUMO}

O esterco de poedeira tem sido utilizado como fertilizante; no entanto, a eficiência deste resíduo em suprir nutrientes às plantas é pouco conhecida. Objetivou-se, neste trabalho, avaliar o efeito de doses deste esterco no suprimento de nitrogênio $(\mathrm{N})$ e no rendimento de grão $(\mathrm{RG})$ da cultura de trigo. As doses testadas foram (base úmida): 2,8; 4,2; 5,6 e 11,2 $\mathrm{t} \mathrm{ha}^{-1}$, comparadas a tratamentos sem esterco (testemunha) mas com ou sem adição de uréia. Os últimos tratamentos foram complementados com fonte mineral de $\mathrm{P}, \mathrm{K}, \mathrm{Ca}$ e $\mathrm{S}$ cujas quantidades foram equivalentes às adicionadas com 5,6 tha-1 de esterco. O experimento foi conduzido em Passo Fundo, Rio Grande do Sul, em um Latossolo Vermelho. O delineamento foi em blocos casualizados (quatro repetições). $\mathrm{O}$ esterco supriu a necessidade de $\mathrm{N}$ do trigo principalmente com a aplicação de 2,8 $\mathrm{t} \mathrm{ha}^{-1}$. Nesta dose e no tratamento com uréia o acúmulo máximo de $\mathrm{N}$ na planta foi no florescimento possibilitando, então, maior mobilização de $\mathrm{N}$ para o grão, acréscimo do número de espigas por área e de grãos por espiga e de RG. A aplicação de 2,8 $\mathrm{t} \mathrm{ha}^{-1}$ de esterco foi suficiente para suprir $\mathrm{N}$ à cultura do trigo proporcionando RG semelhante ao obtido com a aplicação de uréia.

Palavras-chave: Triticum aestivum, adubação, resíduo orgânico

\section{Dose of poultry manure and nitrogen supply for wheat crop}

\begin{abstract}
Poultry manure has been used as fertilizer in grain crops, however, its efficiency to supply nutrients to the plants is not well known. The objective of this study was to evaluate the effect of poultry manure doses on nitrogen $(\mathrm{N})$ supply and on grain yield (GY) of wheat. The doses tested were (wet basis): 2.8, 4.2, 5.6, and $11.2 \mathrm{t} \mathrm{ha}^{-1}$, which were compared to treatments with no manure (control), but with or without urea application. These last treatments were complemented with mineral application of $\mathrm{P}, \mathrm{K}, \mathrm{Ca}$, and $\mathrm{S}$ in amount equivalent applied by $5.6 \mathrm{t} \mathrm{ha}^{-1}$ of manure. The experiment was carried out in Passo Fundo, in the State of Rio Grande do Sul, on a Red Latosol (Typic Haplorthox). A randomized block design was used with four replicates. Poultry manure supplied the amount of $\mathrm{N}$ required by the plants, mainly when the application rate was $2.8 \mathrm{t} \mathrm{ha}^{-1}$. Plants fertilized with this rate and with urea show the highest $\mathrm{N}$ accumulation in anthesis. The application increased $\mathrm{N}$ mobilization to the grains, increased number of spikes per area and grains per spike, and, consequently, GY. Poultry manure applied at $2.8 \mathrm{t} \mathrm{ha}^{-1}$ was enough to supply $\mathrm{N}$ and to increase GY similar to observed by applying $\mathrm{N}$ as urea.
\end{abstract}

Key words: Triticum aestivum, fertilization, organic waste

\footnotetext{
Parte da Dissertação de Mestrado do primeiro autor, Programa de Pós-Graduação em Agronomia (PPGAGRO), Universidade de Passo Fundo - UPF. Financiada pela FAPERGS, Processo 0614463

2 Instituto Nacional de Tecnologia Agropecuária, Estação Experimental de Mercedes. CP 38, CEP 3470, Mercedes, Corrientes, Argentina. Fone: (03773) 421115. E-mail: efigueroa@correo.inta.gov.ar

PPGAGRO/UPF. CP 611, CEP 99001-910, Passo Fundo, RS. Fone: (54) 3316-8151. E-mail: escosteguy@upf.br

Embrapa Trigo, CP 451, CEP 99001-970, Passo Fundo, RS. Fone: (54) 3316-5897. E-mail: siriow@cnpt.embrapa.br
} 


\section{INTRODUÇÃO}

O aumento da produção de ovos tem propiciado um acréscimo no volume de esterco gerado por criadores de aves poedeiras. Com base no plantel mensal de galinhas poedeiras (UBA, 2007) e na quantidade de esterco gerado por esses animais, Santos et al. (2010b) estimaram que cerca de $335.000 \mathrm{t}$ mês ${ }^{-1}$ de esterco foram gerados no País em 2006. Atualmente, esta quantidade deve ter aumentado em função do acréscimo do plantel dessas aves.

O esterco de ave poedeira é considerado fonte importante de nutrientes para os vegetais devido sobretudo ao elevado teor de N (Boateng et al., 2006). A baixa relação C:N do esterco de aves (Santos et al., 2010b) favorece a disponibilidade da maior parte dos nutrientes aplicados às plantas, em especial do $\mathrm{N}$ aspecto que, juntamente com o baixo preço e a alta oferta de esterco, tem motivado a utilização deste resíduo como fertilizante e, sobretudo, fonte de $\mathrm{N}$ às plantas. A expansão desta prática tem sido observada em culturas como trigo e milho, no Rio Grande do Sul, RS; apesar disto, ainda não existem recomendações específicas referentes à pesquisa do Sul do Brasil quanto às quantidades que devem ser aplicadas deste tipo de fertilizante orgânico.

No RS e em Santa Catarina recomenda-se que as doses de esterco sejam estabelecidas de acordo com a quantidade de N, fósforo ou potássio exigida pela cultura com o teor no esterco e no solo e com o índice de disponibilidade desses nutrientes (CQFS-RS/SC, 2004). Contudo, referidos índices ainda não foram estabelecidos para o esterco de ave poedeira sendo conhecidos para os estercos de frango, de suínos e de bovinos. Utilizam-se, por enquanto, os índices sugeridos para outros resíduos orgânicos (excluindo resíduos cujo índice já é conhecido), que correspondem a 0,5 para $\mathrm{N}$; 0,7 para $\mathrm{P}$ e 1,0 para K (CQFS-RS/SC, 2004); assim, os índices dos outros resíduos, como o do esterco de ave poedeira, devem ser validados.

Em adição, são escassos os estudos publicados sobre o efeito de doses do esterco de ave poedeira em culturas agrícolas, sendo a maioria dos trabalhos realizada em condições edafo-climáticas diferente das que predominam nas regiões produtoras de grãos do RS (Nyakatawa et al., 2000; 2001; Balkcom et al., 2003; Sharpe et al., 2004; Endale et al., 2008). Nyakatawa et al. (2000) constataram que a aplicação desse esterco ao solo em quantidade equivalente a $100 \mathrm{~kg} \mathrm{~N} \mathrm{ha}^{-1}$, proporcionou o mesmo rendimento de algodão obtido com essa quantidade de $\mathrm{N}$ aplicada na forma de nitrato de amônio. Já Endale et al. (2008) constataram que, após cinco anos de avaliação, o rendimento de grão (RG) de milho fertilizado com o esterco de poedeira foi $18 \%$ superior que o fertilizado com sulfato ou nitrato de amônio.

Efeitos benéficos da adubação com outros tipos de esterco que não o de ave poedeira têm sido relatados em condições de solo e clima típicos do Brasil (Rocha et al., 2004; Sampaio et al., 2007; Menezes \& Silva, 2008, Santos et al., 2010a), ou de outros países (Loecke et al., 2004; Kiani et al., 2005; Daza-Torres et al., 2008). Referidos trabalhos mostram que a aplicação de esterco de diferentes origens pode aumentar o rendimento das culturas. Por outro lado não há relatos, na literatura, sobre a resposta de culturas de grão à adubação com esterco de ave poedeira, nas condições edafoclimáticas do Brasil. Isto preocupa de vez que a quantidade de esterco a ser adicionada depende, entre outros fatores, da composição deste material, das características do solo, das exigências nutricionais das plantas e das condições climáticas (CQFS-RS/SC, 2004).

Embora a utilização de doses adequadas de estercos contribua para aumentar a eficiência agronômica e econômica das adubações e para evitar eventuais impactos ambientais (CQFS-RS/SC, 2004), esses efeitos ainda não foram avaliados em solos brasileiros adubados com o esterco de poedeira. Objetivou-se, com este trabalho, avaliar o efeito de doses deste esterco no rendimento de grão (RG) e na disponibilidade de nitrogênio $(\mathrm{N})$ à cultura do trigo.

\section{Material e MÉTODOS}

O experimento foi conduzido no Campo Experimental da Faculdade de Agronomia e Medicina Veterinária da Universidade de Passo Fundo, em Passo Fundo, RS, a uma altitude média de $687 \mathrm{~m}$, entre as coordenadas geográficas $28^{\circ}$ 13'37,80" S e 52 23'32,980" W. A precipitação pluvial média anual é de $1.788 \mathrm{~mm}$ e a temperatura média anual é de $17,5^{\circ} \mathrm{C}$ (Cunha, 1997).

O solo da área experimental é um Latossolo Vermelho distrófico húmico, textura argilosa. Antes do início do experimento o solo foi amostrado coletando-se cinco subamostras por parcela, na profundidade de 0 a $10 \mathrm{~cm}$. Efetuouse a análise de solo conforme Tedesco et al. (1995), indicando 23 e $239 \mathrm{mg} \mathrm{dm}^{-3}$ de Pe de $\mathrm{K}$ disponíveis (Mehlich-I); 0,3, 4,0 e 2,3 $\mathrm{cmol}_{\mathrm{c}} \mathrm{dm}^{-3} \mathrm{de} \mathrm{Al}, \mathrm{Ca}$ e Mg e $32 \mathrm{mg} \mathrm{dm}^{-3}$ de $\mathrm{Mn}\left(\mathrm{KCl}, 1 \mathrm{~mol} \mathrm{~L}^{-1}\right) ; 5,4$ e 5,9 de pH em água e índice SMP (suspensão solo:extrator 1:1, 1:1,5; respectivamente), $18 \mathrm{mg} \mathrm{dm}^{-3} \mathrm{de} \mathrm{S}$ disponível $\left(\mathrm{Ca}_{3}\left(\mathrm{PO}_{4}\right)_{2}\right.$,

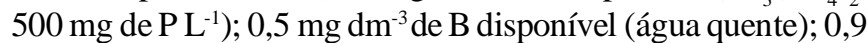
e 1,9 $\mathrm{mg} \mathrm{dm}^{-3} \mathrm{de} \mathrm{Zn}$ e Cu disponíveis ( $\left.\mathrm{HCl}, 0,1 \mathrm{~mol} \mathrm{~L}^{-1}\right) ; 31 \mathrm{~g} \mathrm{dm}^{-}$ ${ }^{3}$ de matéria orgânica (solução sulfocrômica e determinação espectrofotométrica do cromo reduzido) e $450 \mathrm{~g} \mathrm{dm}^{-3}$ de argila (densímetro), respectivamente, em que os resultados indicaram que o solo contém teores elevados de $\mathrm{P}, \mathrm{K}, \mathrm{Ca}, \mathrm{Mg}, \mathrm{S}$ e de micronutrientes, condições em que não há resposta à aplicação desses nutrientes, embora possa ocorrer resposta à adubação nitrogenada (CQFS-RS/SC, 2004); isto possibilitou testar o efeito do $\mathrm{N}$ aplicado com o esterco, isolando-o dos demais nutrientes inerentes à composição deste material orgânico.

O esterco proveio de um aviário de ave poedeira da raça Hy-line e criada em sistema de confinamento, sendo armazenado durante cinco dias em esteiras coletoras de esterco, no interior do aviário, antes do transporte ao local do experimento. Para as análises deste material uma amostra de $2 \mathrm{dm}^{-3}$,composta de cinco subamostras, foi coletada logo assim que o esterco foi descarregado na área experimental.

As análises físico-químicas foram efetuadas conforme Tedesco et al. (1995) e indicaram 8,78 de pH em água, 8,72 de $\mathrm{pH} \mathrm{em} \mathrm{CaCl}_{2} ; 585$ e $615 \mathrm{~g} \mathrm{~kg}^{-1}$ de água avaliada a 65 e $105^{\circ} \mathrm{C}$, respectivamente; $7,33 \mathrm{mS} \mathrm{cm}^{-1}$ de condutividade elétrica; 0,85 $\mathrm{kg} \mathrm{dm}^{-3}$ de densidade e $293 \mathrm{~g} \mathrm{~kg}^{-1}$ de carbono orgânico (WalkleyBlack) na matéria seca. Os teores totais dos demais nutrientes 
obtidos na análise do esterco, expressos em base úmida (585 g água $\mathrm{kg}^{-1}$ ), foram: 28,6 e 2,49 $\mathrm{g} \mathrm{kg}^{-1}$ de $\mathrm{N}$ total e mineral e 35, 20 , 65 e $1,24 \mathrm{~g} \mathrm{~kg}^{-1} \mathrm{de}_{2} \mathrm{O}_{5}$ e $\mathrm{K}_{2} \mathrm{O}, \mathrm{Ca}$ e S, respectivamente. Efetuouse a determinação desses nutrientes com digestão ácida $\left(\mathrm{H}_{2} \mathrm{O}_{2}+\mathrm{H}_{2} \mathrm{SO}_{4}\right)$ e mistura de digestão, exceto o de $\mathrm{N}$ mineral, que foi extraído com $\mathrm{KCl} 1 \mathrm{~mol} \mathrm{~L}^{-1}$.

O delineamento experimental utilizado foi o de blocos casualizados, com quatro repetições. Testaram-se os seguintes tratamentos: 1) testemunha, envolvendo a aplicação de P, K, $\mathrm{Ca}$ e $\mathrm{S}$, em quantidades equivalentes às contidas em $5,6 \mathrm{t} \mathrm{ha}^{-1}$ de esterco e na forma de superfosfato triplo e cloreto de potássio, no sulco de semeadura e de sulfato de cálcio, a lanço; 2) Idem ao tratamento 1 , mais $20 \mathrm{~kg} \mathrm{~N} \mathrm{ha}^{-1}$, aplicado no sulco de semeadura e $60 \mathrm{~kg} \mathrm{~N} \mathrm{ha}^{-1}$, aplicado na superfície do solo, no estádio de afilhamento, ambos na forma de uréia; 3) 2,8 $\mathrm{t} \mathrm{ha}^{-1} \mathrm{de}$ esterco; 4) 4,2 $\mathrm{t} \mathrm{ha}^{-1}$ de esterco; 5) 5,6 $\mathrm{t} \mathrm{ha}^{-1}$ de esterco e 6) 11,2 $\mathrm{t} \mathrm{ha}^{-1}$ de esterco. Os Tratamentos 1 e 2 foram complementados com $\mathrm{P}, \mathrm{K}, \mathrm{Ca}, \mathrm{Mg}$ e S para isolar o efeito da adição desses nutrientes, pois eles estão presentes no esterco. A quantidade adicionada referente aos nutrientes correspondeu às aplicadas com 5,6 t ha-1 (Tratamento 5), já que a quantidade de $\mathrm{N}$ disponível aplicada com essa dose ( $80 \mathrm{~kg} \mathrm{ha}^{-1}$ de $\mathrm{N}$ disponível) corresponde à recomendada para trigo no solo do trabalho, se aplicado o índice de eficiência de 0,5 (CQFS-RS/SC, 2004). Como o teor de micronutrientes do esterco é desprezível os tratamentos utilizados possibilitam comparar a aplicação de $\mathrm{N}$ na forma de esterco com a aplicação de $\mathrm{N}$ na forma de uréia, isolando-se o efeito dos nutrientes aplicados com a dose de $5,6 \mathrm{t} \mathrm{ha}^{-1}$ estabelecida com os atuais critérios da pesquisa regional (CQFS-RS/SC, 2004). Já o tratamento Testemunha sem $\mathrm{N}$ possibilitou isolar o efeito do $\mathrm{N}$ suprido pelo esterco e pela uréia daquele suprido pelo solo e resíduos culturais. Os tratamentos 3, 4, 5 e 6 foram aplicados na superfície do solo, manualmente, a lanço, um dia antes da semeadura.

A cultivar de trigo foi a BRS Camboatá. O espaçamento entre linhas foi de $0,17 \mathrm{~m}$. A densidade de plantas foi de 300 plantas $\mathrm{m}^{-2}$. As parcelas tinham $6 \mathrm{~m}$ de comprimento e $2,23 \mathrm{~m}$ de largura. A área útil de colheita foi de $7,8 \mathrm{~m}^{2}$. A colheita foi realizada manualmente. Avaliaram-se o rendimento, o peso do hectolitro $(\mathrm{PH})$ e os componentes do rendimento dos grãos de trigo, sendo o rendimento e o PH expressos a $130 \mathrm{~g} \mathrm{~kg}^{-1} \mathrm{de}$ umidade. Para avaliação dos componentes do RG e do teor de $\mathrm{N}$ da parte aérea, foram colhidas as plantas contidas em um metro, na linha central das parcelas; em seguida, determinouse o número de grãos espiga ${ }^{-1}$ e se efetuou a contagem do número de espigas. $\mathrm{O}$ número de grãos $\mathrm{m}^{-2}$ foi obtido com a multiplicação do número de grãos espiga ${ }^{-1}$ pelo número de espigas $\mathrm{m}^{-2}$. A massa de mil grãos foi avaliada pela contagem manual de 400 grãos e pela pesagem das amostras secadas a 65 ${ }^{\circ} \mathrm{C}$. Esta temperatura também foi utilizada para secar a parte aérea e os grãos de trigo, na determinação da matéria seca e do teor de $\mathrm{N}$ desses materiais (Tedesco et al., 1995). Para calcular a recuperação pela planta do $\mathrm{N}$ aplicado usou-se o índice de recuperação aparente de N, proposto por Mitchell \& Teel (1977), e se utilizou a equação que consta no rodapé da Tabela 3. Os resultados encontrados foram submetidos à análise de variância e os tratamentos foram comparados com contrastes ortogonais, a $5 \%$ de probabilidade de erro.

\section{RESULTADOS E DISCUSSÃO}

O RG e seus componentes variaram pouco entre os tratamentos (Tabela 1). O Contraste A, que compara o tratamento Testemunha com a média dos tratamentos Testemunha $+\mathrm{N}$ e 2,8 $\mathrm{t} \mathrm{ha}^{-1}$ de esterco, mostra que os valores do RG, do número de espigas por área (NEA) e do número de grãos por espiga (NGE) foram superiores na média desses dois últimos tratamentos (4.194 $\mathrm{kg} \mathrm{ha}^{-1}, 538 \mathrm{e} 32$, respectivamente) em relação ao tratamento Testemunha $\left(3.196 \mathrm{~kg} \mathrm{ha}^{-1}, 402 \mathrm{e} 24\right.$, respectivamente). Como os valores dessas variáveis não diferiram entre os tratamentos comparados no Contraste B, pode-se inferir que o efeito da aplicação de $2,8 \mathrm{t} \mathrm{ha}^{-1}$ equivaleu ao proporcionado pela aplicação de $\mathrm{N}$ na forma de uréia.

O RG das plantas fertilizadas com 2,8 $\mathrm{tha}^{-1}$ de esterco foi superior $\left(4.259 \mathrm{~kg} \mathrm{ha}^{-1}\right)$, em relação à média $\left(3.869 \mathrm{~kg} \mathrm{ha}^{-1}\right)$ das outras doses testadas de esterco $\left(4,2 ; 5,6\right.$ e 11,2 $\left.\mathrm{t} \mathrm{ha}^{-1}\right)$ (Contraste C). Por outro lado, os valores do peso do hectolitro $(\mathrm{PH})$ e da massa de mil grãos (MMG) foram superiores no tratamento Testemunha (Contraste A) e na média das doses mais elevadas de esterco (Contraste C) (Tabela 1) enquanto o contrário foi observado nos resultados do RG, do NEA e do

Tabela 1. Comparação entre tratamentos dos valores de rendimento de grão (RG), peso do hectolitro (PH), número de espiga por área (NEA), massa de mil grãos (MMG) e número de grãos por espiga (NGE) de trigo fertilizado com diferentes doses de esterco de ave poedeira

\begin{tabular}{|c|c|c|c|c|c|}
\hline Contr astes & $\begin{array}{c}\text { RG } \\
\left(\mathrm{kg} \mathrm{ha}^{-1}\right)\end{array}$ & $\begin{array}{c}\mathrm{PH} \\
\left(\mathrm{kg} \mathrm{hL}^{-1}\right)\end{array}$ & $\begin{array}{c}\text { NEA } \\
\text { (espiga } \mathrm{m}^{-2} \text { ) }\end{array}$ & $\begin{array}{l}\text { MMG } \\
(\mathrm{g})\end{array}$ & NGE \\
\hline A) $\mathrm{T}^{1} \mathrm{x}\left(\mathrm{T}+\mathrm{N}^{2} ; 2,8^{3} \mathrm{tha}^{-1}\right)$ & $3.196 \times 4.194 * *$ & $85,6 \times 83,4^{\star *}$ & $402 \times 538^{\star}$ & $33 \times 31^{\star}$ & $24 \times 32^{* *}$ \\
\hline B) $\mathrm{T}+\mathrm{N} \times 2,8 \mathrm{tha}^{-1}$ & $4.129 \times 4.259$ & $83,4 \times 82,8$ & $553 \times 523$ & $31 \times 32$ & $32 \times 32$ \\
\hline C) $2,8 \mathrm{t} \mathrm{ha}^{-1} \times\left(4,2^{4} ; 5,6^{5} ; 11,2^{6} \mathrm{tha} \mathrm{h}^{-1}\right)$ & $4.259 \times 3.869^{*}$ & $82,8 \times 84,8^{\star \star}$ & $523 \times 488$ & $32 \times 34^{*}$ & $32 \times 30$ \\
\hline D) $4,2 \mathrm{t} \mathrm{ha}^{-1} \mathrm{x}\left(5,6 ; 11,2 \mathrm{t} \mathrm{ha}^{-1}\right)$ & $4.071 \times 3.768$ & $84,5 \times 84,9$ & $451 \times 506$ & $34 \times 33$ & $30 \times 30$ \\
\hline E) $5,6 \mathrm{t} \mathrm{ha}^{-1} \times 11,2 \mathrm{t} \mathrm{ha}^{-1}$ & $3.801 \times 3.736$ & $85,2 \times 84,6$ & $518 \times 495$ & $32 \times 34$ & $29 \times 32^{* *}$ \\
\hline CV $(\%)^{\prime}$ & 7,3 & 0,8 & 16,9 & 3,6 & 4,2 \\
\hline
\end{tabular}

${ }^{1}$ Testemunha: P, K, Ca e S de fonte mineral, em quantidade equivalente a contida em 5,6 t ha-1 ${ }^{-1}$ de esterco

${ }^{2}$ dem ao tratamento Testemunha +20 e $60 \mathrm{~kg} \mathrm{~N} \mathrm{ha}^{-1}$, no sulco de semeadura e em cobertura, respectivamente, na forma de uréia

${ }^{3}$ Esterco, 2,8 $\mathrm{t} \mathrm{ha}^{-1}$, correspondendo a $80 \mathrm{~kg} \mathrm{~N} \mathrm{ha}^{-1}$

${ }^{4}$ Esterco, 4,2 tha $^{-1}$, correspondendo a $120 \mathrm{~kg} \mathrm{~N} \mathrm{ha}^{-1}$

${ }^{5}$ Esterco, 5,6 t ha-1, correspondendo a $160 \mathrm{~kg} \mathrm{~N} \mathrm{ha}^{-1}$

${ }^{6}$ Esterco, $11,2 \mathrm{t} \mathrm{ha}^{-1}$, correspondendo a $320 \mathrm{~kg} \mathrm{~N} \mathrm{ha}^{-1}$

${ }^{7}$ Coeficiente de variação

*, **: significativo a 5 e $1 \%$ de probabilidade do erro, respectivamente 
NGE, superiores no tratamento Testemunha $+\mathrm{N}$ e na dose de 2,8 $\mathrm{tha}^{-1}$. Isto se deve, provavelmente, aos menores NGE e NEA observados no tratamento Testemunha, o que possibilitou aproveitamento superior ou redistribuição de fotoassimilados, aumentando o valor do PH e da MMG. Assim, nos tratamentos com maior RG os fotoassimilados foram destinados à formação de mais afilhos ou mais grãos por área, decrescendo o valor do PH e da MMG, constatação que coincide com o relatado em outros trabalhos nos quais foi avaliado o efeito da adubação nitrogenada nos componentes de rendimento (Bredemeier \& Mundstock, 2001; Sangoi et al., 2007; Vieira et al., 2007; Gondim et al., 2008).

Embora o NEA e o NGE não tenham diferido entre as médias dos tratamentos comparados no Contraste $\mathrm{C}$, ocorreu tendência deles serem maiores nas plantas fertilizadas com as menores doses de esterco haja vista que a diferença entre a média desse tratamento e a obtida com as maiores doses de esterco foi significativa a $6,1 \%$ de probabilidade do erro. Assim, entre os componentes de rendimento avaliados, o PH, a MMG e o NGE foram os que mais acompanharam a variação observada no RG, dos tratamentos comparados nos Contrastes A e C (Tabela 1). Tal aspecto também foi observado em outros trabalhos com trigo (Gondim et al., 2008), sendo seu NGE e de outras culturas influenciado pela adubação nitrogenada, principalmente quando o N é aplicado nas etapas iniciais de desenvolvimento das plantas (Bredemeier \& Mundstock, 2001; Sangoi et al., 2007).

A comparação dos contrastes A, B e C evidencia que a aplicação de $\mathrm{N}$ na forma de uréia ou de $2,8 \mathrm{t} \mathrm{ha}^{-1}$ do esterco de poedeira proporcionou os maiores acréscimos no $R G$ de trigo. A eficiência desses tratamentos foi semelhante e o aumento que proporcionou no RG resultou do acréscimo do NEA e do NGE (Contraste A). O RG obtido com a aplicação de $2,8 \mathrm{t} \mathrm{ha}^{-1}$ do esterco de ave poedeira equivaleu ao proporcionado pela aplicação de $\mathrm{N}$ na forma de uréia $($ Testemunha $+\mathrm{N})$, sendo aplicada a mesma quantidade de $\mathrm{N}\left(80 \mathrm{~kg} \mathrm{~N} \mathrm{ha}^{-1}\right)$ nos tratamentos mencionados. Isto evidencia que a eficiência da dose de $2,8 \mathrm{t} \mathrm{ha}^{-1} \mathrm{em}$ suprir $\mathrm{N}$ às plantas de trigo foi de $100 \%$ em relação à eficiência da uréia. Este valor foi expressivamente superior que o atribuído à cama de frango ou a outros resíduos, que é de $50 \%$ (CQFS-RS/SC, 2004). Por outro lado, a eficiência do esterco de ave poedeira foi $20 \%$ superior à estabelecida para dejetos líquidos de suínos, isto é, de $80 \%$ (CQFS-RS/SC, 2004). Desta forma, persistem dúvidas quanto ao índice de eficiência do esterco de ave poedeira.

Os resultados dos Contrastes D e E evidenciam que o RG e seus componentes não diferiram entre as maiores doses de esterco, exceto o NGE, que foi superior com a aplicação da dose de 11,2 t ha-1 de esterco (Contraste E; Tabela 1). Os resultados desses Contrastes comparados com o do Contraste $\mathrm{C}$ indicam que os valores dessas variáveis não diferiram entre os tratamentos com as doses maiores que $2,8 \mathrm{t} \mathrm{ha}^{-1}$ de esterco e a testemunha, haja vista que não houve diferenças entre os tratamentos comparados nos Contrastes D e E, ocorrendo o contrário no Contraste C. É provável que a pouca diferença entre o RG dos tratamentos testados se deva à adequada quantidade e distribuição de chuva, à adequada fertilidade do solo da área experimental, cujos níveis de P, K e de acidez foram corrigidos no cultivo precedente a este experimento; à escarificação do solo efetuada um ano antes da implantação do experimento; ao sistema plantio direto adotado há dez anos na área e à presença de alta quantidade de resíduos culturais na superfície do solo.

Como ocorreu com o RG e seus componentes, o teor e a quantidade de $\mathrm{N}$ total da parte aérea das plantas e dos grãos variaram pouco entre os tratamentos. No estádio de afilhamento e nos grãos os teores de $\mathrm{N}$ não foram influenciados pelos tratamentos testados (Tabelas 2 e 3 , respectivamente) o que se devem, sem dúvida, à menor quantidade de $\mathrm{N}$ demandada pela planta no estádio de afilhamento e ao suprimento de $\mathrm{N}$ pelo solo e resíduos culturais da soja. Considerando que a contribuição desses resíduos pode equivaler a $1 \mathrm{~kg}$ de $\mathrm{N}$ por $60 \mathrm{~kg}$ de grãos colhidos (Halvorson et al., 1987), cerca de $80 \mathrm{~kg} \mathrm{~N} \mathrm{ha}^{-1}$ podem ter sido fornecidos, desta forma, à cultura do trigo. No estádio de afilhamento verificou-se, também, que a diferença entre o teor de $\mathrm{N}$ das plantas dos tratamentos comparados no Contraste $\mathrm{B}$ foi significativa a $6,8 \%$ de probabilidade do erro próximo, portanto, aos $5 \%$ considerados no trabalho, evidenciando que

Tabela 2. Comparação entre tratamentos dos teores e quantidades de nitrogênio $(\mathrm{N})$, da matéria seca (MS) da parte aérea e do índice de recuperação aparente deste nutriente (IRAN), no estádio de florescimento, em trigo fertilizado com diferentes doses de esterco de ave poedeira

\begin{tabular}{|c|c|c|c|c|c|}
\hline \multirow{3}{*}{ Contrastes } & \multirow{3}{*}{$\begin{array}{c}\text { Afilhamento } \\
\text { (\%) }\end{array}$} & \multicolumn{3}{|c|}{ Florescimento } & \multirow{3}{*}{$\begin{array}{c}\text { IRAN }^{8} \\
(\%)\end{array}$} \\
\hline & & \multirow{2}{*}{$\begin{array}{l}\mathbf{N} \\
\%\end{array}$} & MS & $\mathbf{N}$ & \\
\hline & & & \multicolumn{2}{|c|}{$\left(\mathrm{kg} \mathrm{ha}^{-1}\right)$} & \\
\hline A) $T^{1} x\left(T+N^{2} ; 2,8^{3} t_{h a}^{-1}\right)$ & $4,4 \times 4,9$ & $2,6 \times 3,3 * *$ & $2.113,1 \times 4.031,5^{\star * *}$ & $55,2 \times 133,5^{\star \star}$ & - \\
\hline B) $\mathrm{T}+\mathrm{N} \times 2,8 \mathrm{tha}^{-1}$ & $5,3 \times 4,6$ & $3,4 \times 3,3$ & $4.153,9 \times 3.905,5$ & $139,6 \times 127,5$ & $105,5 \times 90,4$ \\
\hline C) $2,8 \mathrm{tha}^{-1} \times\left(4,2^{4} ; 5,6^{5} ; 11,2^{6} \mathrm{tha}^{-1}\right)$ & $4,6 \times 4,8$ & $3,3 \times 3,3$ & $3.905,5 \times 3.672,7$ & $127,5 \times 121,3$ & $90,4 \times 35,1^{\star \star}$ \\
\hline D) $4,2 \mathrm{t} \mathrm{ha}^{-1} \times\left(5,6 ; 11,2 \mathrm{t} \mathrm{ha}^{-1}\right)$ & $4,8 \times 4,8$ & $3,0 \times 3,5^{\star}$ & $3.403,4 \times 3.788,8$ & $101,5 \times 131,1^{\star \star}$ & $38,6 \times 33,3$ \\
\hline E) $5,6 \mathrm{tha}^{-1} \times 11,2 \mathrm{tha}^{-1}$ & $4,8 \times 4,7$ & $3,3 \times 3,6$ & $3.505,3 \times 4.051,5$ & $116,7 \times 145,6^{*}$ & $38,5 \times 28,2$ \\
\hline $\mathrm{CV}(\%)^{7}$ & 10,9 & 8,4 & 13,2 & 12,6 & 22,6 \\
\hline
\end{tabular}

${ }^{1}$ Testemunha: $\mathrm{P}, \mathrm{K}, \mathrm{Ca}$ e $\mathrm{S}$ de fonte mineral, em quantidade equivalente à contida em $5,6 \mathrm{t} \mathrm{ha}{ }^{-1}$ de esterco

${ }^{2}$ Idem ao tratamento Testemunha +20 e $60 \mathrm{~kg} \mathrm{~N} \mathrm{ha}^{-1}$, no sulco de semeadura e em cobertura, respectivamente, na forma de uréia

${ }^{3}$ Esterco, 2,8 $\mathrm{t} \mathrm{ha}^{-1}$, correspondendo a $80 \mathrm{~kg} \mathrm{~N} \mathrm{ha}^{-1}$

${ }^{4}$ Esterco, 4,2 tha $^{-1}$, correspondendo a $120 \mathrm{~kg} \mathrm{~N} \mathrm{ha}^{-1}$

${ }^{5}$ Esterco, 5,6 tha-1 ${ }^{-1}$ correspondendo a $160 \mathrm{~kg} \mathrm{~N} \mathrm{ha}^{-1}$

${ }^{6}$ Esterco, 11,2 t ha-1 ${ }^{-1}$ correspondendo a $320 \mathrm{~kg} \mathrm{~N} \mathrm{ha}^{-1}$

${ }^{7}$ Coeficiente de variação

${ }^{8}$ IRAN $=[(\mathrm{N}$ tratamento $-\mathrm{N}$ testemunha $) / \mathrm{N}$ aplicado $] \times 100$

${ }^{\star},{ }^{\star \star},{ }^{* \star \star}$ : significativo a 5,1 e $0,1 \%$ de probabilidade do erro, respectivamente 
Tabela 3. Comparação entre tratamentos dos valores de nitrogênio $(\mathrm{N})$ e do índice de recuperação aparente desse nutriente (IRAN), em grãos de trigo fertilizado com diferentes doses de esterco de ave poedeira

\begin{tabular}{|c|c|c|c|}
\hline \multirow{2}{*}{ Contr astes } & \multicolumn{2}{|c|}{ Nitrogênio do grão } & \multirow{2}{*}{$\begin{array}{c}\text { IRAN }^{8} \\
(\%)\end{array}$} \\
\hline & (\%) & $\left(\mathrm{kg} \mathrm{ha}^{-1}\right)$ & \\
\hline A) $T^{1} x\left(T+N^{2} ; 2,8^{3} t h a^{-1}\right)$ & $1,6 \times 2,0$ & $51,1 \times 81,8^{* *}$ & 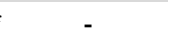 \\
\hline B) $\mathrm{T}+\mathrm{N} \times 2,8 \mathrm{tha}^{-1}$ & $2,0 \times 1,9$ & $82,6 \times 80,9$ & $39,3 \times 37,3$ \\
\hline C) $2,8 \mathrm{t} \mathrm{ha}^{-1} \times\left(4,2^{4} ; 5,6^{\mathrm{b}} ; 11,2^{6} \mathrm{tha} \mathrm{ha}^{-1}\right)$ & $1,9 \times 1,8$ & $80,9 \times 69,6$ & $37,3 \times 11,0$ ** \\
\hline D) $4,2 \mathrm{t} \mathrm{ha}^{-1} \times\left(5,6 ; 11,2 \mathrm{t} \mathrm{ha}^{-1}\right)$ & $1,8 \times 1,8$ & $73,3 \times 67,8$ & $18,5 \times 7,3$ \\
\hline E) $5,6 \mathrm{t} \mathrm{ha}^{-1} \times 11,2 \mathrm{t} \mathrm{ha}^{-1}$ & $1,7 \times 1,9$ & $64,6 \times 71,0$ & $8,4 \times 6,2$ \\
\hline CV $(\%)^{7}$ & & & 42,4 \\
\hline \multicolumn{4}{|c|}{ 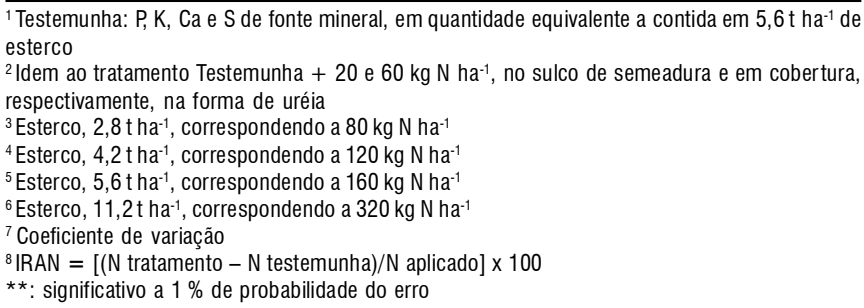 } \\
\hline
\end{tabular}

o teor de $\mathrm{N}$ das plantas do tratamento Testemunha $+\mathrm{N}(5,3 \%)$ tendeu a ser maior que o das plantas fertilizadas com $2,8 \mathrm{t} \mathrm{ha}^{-1} \mathrm{de}$ esterco (4,6\%) (Tabela 2).

Por outro lado, no estádio de florescimento houve diferenças entre as médias dos tratamentos comparados nos Contrastes A e D. Nesse estádio o teor de $\mathrm{N}$ na planta foi maior na média dos tratamentos Testemunha $+\mathrm{N}$ e 2,8 $\mathrm{t} \mathrm{ha}^{-1}$ esterco, em relação ao tratamento Testemunha (Contraste A), e na média das doses mais altas de esterco (Contraste D) (Tabela 2).

$\mathrm{O}$ maior teor de $\mathrm{N}$ proporcionado pelos tratamentos Testemunha $+\mathrm{Ne} 2,8 \mathrm{t} \mathrm{ha}^{-1}$ esterco contribuíram para aumentar o NEA, o NGE e, consequentemente, o RG (Tabela 1). No florescimento o tratamento com 11,2 $\mathrm{t} \mathrm{ha}^{-1}$ de esterco proporcionou maior quantidade de $\mathrm{N}$ acumulada nas plantas (Contraste E; Tabela 2), o que contribuiu, possivelmente, para o maior NGE obtido com esta dose em relação à de 5,6 $\mathrm{t} \mathrm{ha}^{-1}$ (Contraste E; Tabela 1). Referidos efeitos estão relacionados com o maior acúmulo de biomassa proporcionado pelo maior teor de $\mathrm{N}$ das plantas e a maior capacidade de transferi-la às estruturas reprodutivas em preflorescimento (Gondim et al., 2008). Já os maiores valores do NEA e do NGE influenciaram positivamente o RG, que varia em função principalmente do produto desses componentes e da MMG, sendo que esses três fatores variam, independentemente um do outro (Vieira et al., 2007).

Da mesma forma que o observado em relação ao NGE comparado no Contraste C (Tabela 1), a quantidade de $\mathrm{N}$ dos grãos também tendeu a ser maior nas plantas fertilizadas com $2,8 \mathrm{t} \mathrm{ha}^{-1}$ de esterco $\left(80,9 \mathrm{~kg} \mathrm{ha}^{-1}\right)$, que com a média das demais doses $\left(69,6 \mathrm{~kg} \mathrm{ha}^{-1}\right)($ Contraste $\mathrm{C}$; Tabela 3$)$, já que a diferença entre os tratamentos comparados neste contraste foi significativa a 9,6\% de probabilidade do erro. Como comentado em relação ao NGE, esta tendência acompanhou o maior valor da MMG e, em consequência, o maior RG proporcionado pela aplicação de 2,8 $\mathrm{t} \mathrm{ha}^{-1}$ de esterco (Contraste C, Tabela 1).

Os maiores índices de recuperação aparente de $\mathrm{N}$ (IRAN) foram proporcionados pelos tratamentos Testemunha $+\mathrm{N}$ e $2,8 \mathrm{t} \mathrm{ha}^{-1}$ de esterco (Tabelas 2 e 3 ). No estádio de florescimento este índice foi de $105,5 \%$, no tratamento Testemunha $+\mathrm{N}$ e de $90,4 \%$, com $2,8 \mathrm{t} \mathrm{ha}^{-1}$ de esterco, sem diferir entre esses tratamentos (Contraste B; Tabela 2). Tais valores foram maiores que o dobro das outras doses (Contraste C; Tabela 2); já os valores dos IRAN calculados com o $\mathrm{N}$ do grão decresceram em relação ao do florescimento da planta, fato observado em todos os tratamentos (Tabela 3). Os maiores valores de IRAN no grão foram 39,3\% (Testemunha $+\mathrm{N})$ e $37,3 \%\left(2,8 \mathrm{t} \mathrm{ha}^{-1} \mathrm{de}\right.$ esterco), enquanto nos demais tratamentos os valores foram expressivamente menores, variando de 6,2 a 18,5\% (Tabela 3 ). Nota-se, comparando-se esses resultados com os da Tabela 2, que a maior quantidade de $\mathrm{N}$ observada no florescimento das plantas fertilizadas com 11,2 $\mathrm{t} \mathrm{ha}^{-1}$ não foi suficiente para proporcionar que o $\mathrm{RG}$ deste tratamento fosse superior que os dos tratamentos Testemunha $+\mathrm{N}$ e 2,8 $\mathrm{t} \mathrm{ha}^{-1}$ de esterco. A maior eficiência desses últimos condiz com os maiores valores de NEA, NGE e quantidade de N nos grãos (Tabelas 1 e 3). Os maiores IRAN desses tratamentos também explicam os maiores teores (florescimento) e quantidades extraídas de $\mathrm{N}$ em relação ao Tratamento testemunha sem N (Contraste A; Tabelas 2 e 3).

Como constatado com as demais variáveis avaliadas, os teores e as quantidades extraídas dos demais nutrientes analisados nos grãos variaram pouco entre as doses testadas (Tabelas 4 a 6).

Tabela 4. Comparação entre tratamentos dos valores de fósforo, potássio e cálcio em grão de trigo fertilizado com diferentes doses de esterco de ave poedeira

\begin{tabular}{|c|c|c|c|c|c|c|}
\hline \multirow{2}{*}{ Contrastes } & \multicolumn{2}{|c|}{ Fósfor 0} & \multicolumn{2}{|c|}{ Potássio } & \multicolumn{2}{|c|}{ Cálcio } \\
\hline & $(\%)$ & $\left(\mathrm{kg} \mathrm{ha}^{-1}\right)$ & (\%) & $\left(\mathrm{kg} \mathrm{ha}^{-1}\right)$ & $(\%)$ & $\left(\mathrm{kg} \mathrm{ha}^{-1}\right)$ \\
\hline A) $T^{1} \times\left(T+N^{2} ; 2,8^{3} t h a^{-1}\right)$ & $0,39 \times 0,42$ & $12,5 \times 17,6^{\star}$ & $0,35 \times 0,37$ & $11,2 \times 15,5^{\star}$ & $0,05 \times 0,04$ & $1,6 \times 1,7$ \\
\hline B) $\mathrm{T}+\mathrm{N} \times 2,8 \mathrm{t} \mathrm{ha}^{-1}$ & $0,42 \times 0,43$ & $17,3 \times 18,3$ & $0,37 \times 0,36$ & $15,6 \times 15,3$ & $0,04 \times 0,04$ & $1,7 \times 1,7$ \\
\hline C) $2,8 \mathrm{tha}^{-1} \times\left(4,2^{4} ; 5,6^{5} ; 11,2^{6} \mathrm{tha}^{-1}\right)$ & $0,43 \times 0,50$ & $18,3 \times 19,3$ & $0,36 \times 0,40$ & $15,3 \times 15,5$ & $0,04 \times 0,04$ & $1,7 \times 1,5$ \\
\hline D) $4,2 \mathrm{tha}^{-1} \times\left(5,6 ; 11,2 \mathrm{t} \mathrm{ha}^{-1}\right)$ & $0,47 \times 0,52$ & $19,1 \times 19,4$ & $0,36 \times 0,42$ & $14,7 \times 15,8$ & $0,03 \times 0,04$ & $1,2 \times 1,5$ \\
\hline E) $5,6 \mathrm{t} \mathrm{ha}^{-1} \times 11,2 \mathrm{t} \mathrm{ha}^{-1}$ & $0,49 \times 0,54$ & $18,6 \times 20,2$ & $0,38 \times 0,45$ & $14,4 \times 16,8$ & $0,04 \times 0,04$ & $1,5 \times 1,5$ \\
\hline CV $(\%)^{7}$ & 20,4 & 19,5 & 20,2 & 21,0 & 28,8 & 29,2 \\
\hline
\end{tabular}

${ }^{1}$ Testemunha: $\mathrm{P}, \mathrm{K}, \mathrm{Ca}$ e $\mathrm{S}$ de fonte mineral, em quantidade equivalente à contida em $5,6 \mathrm{t}$ ha ${ }^{-1}$ de esterco

${ }^{2}$ Idem ao tratamento Testemunha +20 e $60 \mathrm{~kg} \mathrm{~N} \mathrm{ha}^{-1}$, no sulco de semeadura e em cobertura, respectivamente, na forma de uréia

${ }^{3}$ Esterco, 2,8 $\mathrm{tha}^{-1}$, correspondendo a $80 \mathrm{~kg} \mathrm{~N} \mathrm{ha}^{-1}$

${ }^{4}$ Esterco, 4,2 tha $^{-1}$, correspondendo a $120 \mathrm{~kg} \mathrm{~N}^{5} \mathrm{a}^{-1}$

${ }^{5}$ Esterco, 5,6 t ha-1, correspondendo a $160 \mathrm{~kg} \mathrm{~N} \mathrm{ha}^{-1}$

${ }^{6}$ Esterco, $11,2 \mathrm{t} \mathrm{ha}^{-1}$, correspondendo a $320 \mathrm{~kg} \mathrm{~N} \mathrm{ha}^{-1}$

${ }^{7}$ Coeficiente de variação

*: significativo a $5 \%$ de probabilidade do erro 
Tabela 5. Comparação entre tratamentos dos valores de magnésio, de enxofre e de zinco de grãos de trigo fertilizado com diferentes doses de esterco de ave poedeira

\begin{tabular}{|c|c|c|c|c|c|c|}
\hline \multirow{2}{*}{ Contr astes } & \multicolumn{2}{|c|}{ Magnésio } & \multicolumn{2}{|c|}{ Enxofre } & \multicolumn{2}{|c|}{ Zinco } \\
\hline & $(\%)$ & $\left(\mathrm{kg} \mathrm{ha}^{-1}\right)$ & $(\%)$ & $\left(k_{g} h a^{-1}\right)$ & $\left(\mathrm{mg} \mathrm{kg}^{-1}\right)$ & $\left(g\right.$ ha $\left.^{-1}\right)$ \\
\hline A) $\mathrm{T}^{1} \mathrm{x}\left(\mathrm{T}+\mathrm{N}^{2} ; 2,8^{3} \mathrm{tha}^{-1}\right)$ & $0,12 \times 0,10$ & $3,8 \times 4,2$ & $0,13 \times 0,14$ & $4,2 \times 5,9^{\star}$ & $28,7 \times 25,8$ & $91,7 \times 108,2^{\star *}$ \\
\hline B) $\mathrm{T}+\mathrm{N} \times 2,8 \mathrm{tha}^{-1}$ & $0,10 \times 0,10$ & $4,1 \times 4,3$ & $0,13 \times 0,14$ & $5,4 \times 6,0$ & $27,2 \times 24,5$ & $112,3 \times 104,3$ \\
\hline C) $2,8 \mathrm{t} \mathrm{ha}^{-1} \mathrm{x}\left(4,2^{4} ; 5,6^{5} ; 11,2^{6} \mathrm{t} \mathrm{ha}{ }^{-1}\right)$ & $0,10 \times 0,12$ & $4,3 \times 4,6$ & $0,14 \times 0,12$ & $6,0 \times 4,6^{*}$ & $24,5 \times 25,2$ & $104,3 \times 97,5$ \\
\hline D) $4,2 \mathrm{t} \mathrm{ha}{ }^{-1} \times\left(, 6 ; 11,2 \mathrm{t} \mathrm{ha}^{-1}\right)$ & $0,11 \times 0,13$ & $4,5 \times 4,9$ & $0,12 \times 0,12$ & $4,9 \times 4,5$ & $25,8 \times 24,8$ & $105,0 \times 93,4$ \\
\hline E) $5,6 \mathrm{t} \mathrm{ha}^{-1} \times 11,2 \mathrm{t} \mathrm{ha}^{-1}$ & $0,12 \times 0,13$ & $4,6 \times 4,9$ & $0,14 \times 0,10$ & $5,3 \times 3,7$ & $25,6 \times 24,1$ & $97,3 \times 90,0$ \\
\hline CV $(\%)^{7}$ & 29,1 & 29,9 & 24,5 & 23,9 & 9,5 & 10,5 \\
\hline
\end{tabular}

${ }^{1}$ Testemunha: $\mathrm{P}, \mathrm{K}, \mathrm{Ca}$ e $\mathrm{S}$ de fonte mineral, em quantidade equivalente à contida em $5,6 \mathrm{t}$ ha ${ }^{-1}$ de esterco

${ }^{2}$ Idem ao tratamento Testemunha +20 e $60 \mathrm{~kg} \mathrm{~N} \mathrm{ha}^{-1}$, no sulco de semeadura e em cobertura, respectivamente, na forma de uréia

${ }^{3}$ Esterco, 2,8 $\mathrm{tha}^{-1}$, correspondendo a $80 \mathrm{~kg} \mathrm{~N} \mathrm{ha}^{-1}$

${ }^{4}$ Esterco, 4,2 $\mathrm{tha}^{-1}$, correspondendo a $120 \mathrm{~kg} \mathrm{~N} \mathrm{ha}^{-1}$

${ }^{5}$ Esterco, 5,6 tha-1 ${ }^{-1}$ correspondendo a $160 \mathrm{~kg} \mathrm{~N} \mathrm{ha}^{-1}$

${ }^{6}$ Esterco, 11,2 $\mathrm{t} \mathrm{ha}^{-1}$, correspondendo a $320 \mathrm{~kg} \mathrm{~N} \mathrm{ha}^{-1}$

${ }^{7}$ Coeficiente de variação

*, **: significativo a 5 e $1 \%$ de probabilidade do erro, respectivamente

Tabela 6. Comparação entre tratamentos dos valores de cobre, de manganês e de ferro em grãos de trigo fertilizado com diferentes doses de esterco de ave poedeira

\begin{tabular}{|c|c|c|c|c|c|c|}
\hline \multirow{2}{*}{ Contr astes } & \multicolumn{2}{|c|}{ Cobre } & \multicolumn{2}{|c|}{ Manganês } & \multicolumn{2}{|c|}{ Ferro } \\
\hline & $\left(\mathrm{mg} \mathrm{kg}^{-1}\right)$ & $\left(\mathrm{gha}^{-1}\right)$ & $\left(\mathrm{mg} \mathrm{kg}^{-1}\right)$ & $\left(g_{h a}{ }^{-1}\right)$ & $\left(\mathrm{mg} \mathrm{kg}^{-1}\right)$ & $\left(g \mathrm{ha}^{-1}\right)$ \\
\hline A) $T^{1} x\left(T+N^{2} ; 2,8^{3} t h a^{-1}\right)$ & $6,2 \times 6,0$ & $19,9 \times 25,2$ & $28,8 \times 32,3$ & $92,0 \times 135,5^{\star *}$ & $68,9 \times 47,0$ & $220,2 \times 197,1$ \\
\hline B) $\mathrm{T}+\mathrm{N} \times 2,8 \mathrm{tha}^{-1}$ & $6,1 \times 5,9$ & $25,1 \times 25,4$ & $34,7 \times 29,9^{*}$ & $143,3 \times 127,3$ & $35,8 \times 58,2$ & $147,8 \times 247,9$ \\
\hline C) $2,8 \mathrm{t} \mathrm{ha}^{-1} \times\left(4,2^{4} ; 5,6^{5} ; 11,2^{6} \mathrm{tha}^{-1}\right)$ & $5,9 \times 6,2$ & $25,4 \times 24,0$ & $29,9 \times 27,0$ & $127,3 \times 104,5^{\star *}$ & $58,2 \times 28,1^{*}$ & $247,9 \times 108,7^{* *}$ \\
\hline D) $4,2 \mathrm{t} \mathrm{ha}^{-1} \times\left(5,6 ; 11,2 \mathrm{tha}^{-1}\right)$ & $6,2 \times 6,2$ & $25,2 \times 23,4$ & $26,6 \times 27,2$ & $108,3 \times 102,5$ & $20,2 \times 32,0$ & $82,2 \times 120,6$ \\
\hline $\begin{array}{l}\text { E) } 5,6 \mathrm{t} \mathrm{ha}^{-1} \times 11,2 \mathrm{tha}^{-1} \\
\text { CV }(\%)^{7}\end{array}$ & $\begin{array}{c}6,5 \times 5,9^{*} \\
3,6\end{array}$ & $\begin{array}{c}24,7 \times 22,2^{\star} \\
6,9\end{array}$ & $\begin{array}{c}27,3 \times 27,1 \\
9,6\end{array}$ & $\begin{array}{c}103,8 \times 101,2 \\
10,8\end{array}$ & $\begin{array}{c}22,1 \times 42,0 \\
46,3\end{array}$ & $\begin{array}{c}84,0 \times 156,9 \\
50,3\end{array}$ \\
\hline
\end{tabular}

${ }^{1}$ Testemunha: $\mathrm{P}, \mathrm{K}, \mathrm{Ca}$ e $\mathrm{S}$ de fonte mineral, em quantidade equivalente à contida em $5,6 \mathrm{t} \mathrm{ha}^{-1} \mathrm{de}$ esterco

${ }^{2}$ Idem ao tratamento Testemunha +20 e $60 \mathrm{~kg} \mathrm{~N} \mathrm{ha}^{-1}$, no sulco de semeadura e em cobertura, respectivamente, na forma de uréia

${ }^{3}$ Esterco, 2,8 $\mathrm{tha}^{-1}$, correspondendo a $80 \mathrm{~kg} \mathrm{~N} \mathrm{ha}^{-1}$

${ }^{4}$ Esterco, 4,2 tha $^{-1}$, correspondendo a $120 \mathrm{~kg} \mathrm{~N} \mathrm{ha}^{-1}$

${ }^{5}$ Esterco, 5,6 tha- ${ }^{-1}$, correspondendo a $160 \mathrm{~kg} \mathrm{~N} \mathrm{ha}^{-1}$

${ }^{6}$ Esterco, 11,2 $\mathrm{t} \mathrm{ha}^{-1}$, correspondendo a $320 \mathrm{~kg} \mathrm{~N} \mathrm{ha}^{-1}$

${ }^{7}$ Coeficiente de variação

*,**: significativo a 5 e $1 \%$ de probabilidade do erro, respectivamente

\section{CONCLUSÕES}

1. A aplicação de $2,8 \mathrm{t} \mathrm{ha}^{-1}$ de esterco de ave poedeira foi suficiente para suprir a cultura do trigo, proporcionando $R G$ semelhante ao obtido com a aplicação deste nutriente na forma de uréia.

2. Doses maiores que $2,8 \mathrm{tha}^{-1}$ de esterco de ave poedeira reduzem o $\mathrm{RG}$ de trigo.

\section{LITERATURA CITADA}

Balkcom, K. S.; Adams, J. F.; Hartzog, D. L. Peanut yield response to poultry litter and municipal sludge application. Communication of Soil Science and Plant Analyses, v.152, p.118-33, 2003.

Boateng, S.A.; Zickermann, J.; Kornahrens, M. Poultry manure effect on growth and yield of maize. West Africa Journal of Applied Ecology, v.9, p.1-11, 2006.

Bredemeier, C.; Mundstock, C. M. Estádios fenológicos do trigo para a adubação nitrogenada em cobertura. Revista Brasileira de Ciência do Solo, v.25, p.317-323, 2001.

CQFS-RS/SC - Comissão de Química e Fertilidade do Solo. Manual de adubação e de calagem para os Estados do Rio Grande do Sul e de Santa Catarina. Porto Alegre: SBCS, 2004. 400p.
Cunha, G. R. Meteorologia: Fatos \& mitos. Passo Fundo: Embrapa Trigo, 1997. 267p.

Daza-Torres, M. C.; Alvarez-Herrera, J. G.; Camacho-Tamayo, J. H. Aplicación de materiales orgánicos e inorgánicos en la adsorción de fósforo en un Oxisol. Revista Brasileira Engenharia Agrícola e Ambiental, v.12, p.451-457, 2008.

Endale, D. M.; Shomberg, H. H.; Fisher, D. S.; Jenkins, M. B.; Sharpe, R. R.; Cabrera, M. L. No-till corn productivity in a Southeastern United States ultisol amended with poultry litter. Agronomy Journal, v.10, p.1401-1408, 2008.

Gondim, T. C. de O.; Rocha, V. S.; Sediyama, C. S.; Miranda, G. V. Análise de trilha para componentes do rendimento e caracteres agronômicos de trigo sob desfolha. Pesquisa Agropecuária Brasileira, v.43, p.487-493, 2008.

Halvorson, A. D; Alley, M. M.; Murphy, L. S. Nutrient requirements and fertilizer use. In: HEYNE, E. G. Wheat and wheat improvement. 2.ed. Madison: ASA-CSSA-SSSA, p.345-383. 1987.

Kiani, M. J.; Abbasi, M. K.; Rahim, N. Use of organic manure with mineral $\mathrm{N}$ fertilizer increases wheat yield at Rawalakot Azad Jammu and Kashmir. Archives of Agronomy and Soil Science, v.51, p.299-309, 2005.

Loecke, T. D.; Liebman, M.; Cambardella, C. A.; Richard, T. L. Corn response to composting and time of application of solid swine manure. Agronomy Journal, v.96, p.214-223, 2004. 
Menezes, R. S. C.; Silva. T. O. da. Mudanças na fertilidade de um Neossolo Regolítico após seis anos de adubação orgânica. Revista Brasileira de Engenharia Agrícola e Ambiental, v.12, p.251-257, 2008.

Mitchell, W. H.; Teel, M. R. Winter-annual cover crops for no-tillage corn production. Agronomy Journal, v.69, p.569-573, 1977.

Nyakatawa, E. Z.; Reddy, K. C.; Mays, D. A. Tillage, cover cropping, and poultry litter effects on cotton: II. Growth and yield parameters. Agronomy Journal, v.92, p.1000-1007, 2000.

Nyakatawa, E. Z.; Reddy, K. C.; Sistani, K. R. Tillage, cover cropping, and poultry litter effects on selected soil chemical properties. Soil \& Tillage Research, v.58, p.69-79, 2001.

Rocha, G. N.; Gonçalves, J. L. M.; Moura, I. M. Mudanças da fertilidade do solo e crescimento de um povoamento de Eucalyptus grandis fertilizado com biossólido. Revista Brasileira de Ciência do Solo, v.28, p.623-639, 2004.

Sampaio, E. V. de S. B.; Oliveira, N. M. B. de; Nascimento, P. R. F. do. Eficiência da adubação orgânica com esterco bovino e com Egeria densa. Revista Brasileira de Ciência do Solo, v.31, p.995-1002, 2007.

Sangoi, L.; Berns, A. C.; Almeida, M. L. de; Zanin, C. G.; Schweitzer, C. Características agronômicas de cultivares de trigo em resposta à época da adubação nitrogenada de cobertura. Ciência Rural, v.37, p.1564-1570, 2007.
Santos, A. F. dos; Menezes, R. S. C.; Fraga, V. S.; Pérez-Marin, A. M. Efeito residual da adubação orgânica sobre a produtividade de milho em sistema agroflorestal. Revista Brasileira de Engenharia Agrícola e Ambiental, v.14, p.12671272, 2010a.

Santos, F. G.; Escosteguy, P. A. V.; Rodrigues, L. B. Qualidade de compostos de esterco de ave poedeira submetido a dois tipos de tratamento de compostagem. Revista Brasileira de Engenharia Agrícola e Ambiental, v.14, p.1101-1108, 2010 b.

Sharpe, R. R.; Schomberg, H. H.; Harper, L. A.; Endale, D. M.; Jenkins, M. B.; Franzluebbers, A. J. Atmospheric pollutants and trace gases. Journal of Environmental Quality, v.33, p.1183-1188, 2004.

Tedesco, M. J.; Gianello, C.; Bissani, C. A.; Bohnen, H. E.; Volkweiss, S. J. Análises de solo, plantas e outros materiais. 2.ed. Porto Alegre: UFRGS, 1995. 188p. Boletim técnico, 5

UBA - União Brasileira de Avicultura. São Paulo: UBA, 2007. 82p. Relatório Anual 2006/2007.

Vieira, E. A.; Carvalho, F. I. de; Oliveira, A. C. de; Martins, L. F.; Benin, G.; Silva, J. A. G. da; Coimbra, J.; Martins, A. F.; Carvalho, M. F. de; Ribeiro, G. Análise de trilha entre os componentes primários e secundários do rendimento de grãos em trigo. Revista Brasileira de Agrociência, v.13, p.169$174,2007$. 